Revue d'histoire de l'enfance « irrégulière »

Le Temps de l'histoire

Hors-série | 2001

Histoire et justice, panorama de la recherche

\title{
Le débat de 1908 sur la peine de mort
}

\author{
Gérard Baal
}

\section{OpenEdition}

Journals

Édition électronique

URL : http://journals.openedition.org/rhei/431

DOI : 10.4000/rhei.431

ISBN : 978-2-7535-1641-0

ISSN : 1777-540X

Éditeur

Presses universitaires de Rennes

Édition imprimée

Date de publication : 15 novembre 2001

Pagination : 113-126

ISSN : 1287-2431

Référence électronique

Gérard Baal, «Le débat de 1908 sur la peine de mort », Revue d'histoire de l'enfance « irrégulière » [En ligne], Hors-série | 2001, mis en ligne le 31 mai 2007, consulté le 07 mai 2019. URL : http:// journals.openedition.org/rhei/431 ; DOI : 10.4000/rhei.431 


\section{Le débat de 1908 sur la peine de mort Gérard Ball $^{(1)}$}

Pourquoi revenir sur ces années 1906-1908 où la peine de mort a failli disparaitre ? D'abord parce que l'échec des abolitionnistes peut surprendre, au lendemain d'une grande victoire électorale de la gauche. La France républicaine se vante de montrer les voies du progrès au monde. En la circonstance, elle renonce à suivre l'exemple de plusieurs monarchies voisines. ${ }^{(2)}$ La portée de l'échec de 1908 est considérable, puisqu'il n'y aura plus de débat parlementaire de ce genre avant 1981.

Cette communication sera essentiellement une analyse du débat de 1908 à la Chambre des députés. ${ }^{(3)}$ Nous sommes à l'âge d'or de l'éloquence parlementaire, et la fête oratoire du Palais Bourbon est un important révélateur de l'état des mentalités. Le contexte, et notamment le rôle de la presse dans le développement d'un état d'esprit "sécuritaire", a été fort bien étudié dans des ouvrages récents de Robert $\mathrm{Nye}^{(4)}$ et de Dominique Kalifa. ${ }^{(5)}$

\section{"L'insécurité est à la mode ${ }^{(6)}$ "}

En 1906, la guillotine semble condamnée. Elle va cesser de fonctionner pendant trois ans, le président de la République, Fallières, graciant systématiquement les condamnés. Le président du Conseil, Clemenceau, est abolitionniste. La commission du budget a supprimé les crédits destinés à rémunérer l'exécuteur des hautes œuvres mais, le 30 novembre, le gouvernement demande à la Chambre de les rétablir, ne voulant pas que l'abolition se fasse de façon détournée. Il dépose immédiatement un projet de loi remplaçant la peine de mort par l'internement à perpétuité, précédé de six ans d'encellulement. Le vote du 30 novembre a été acquis à une courte majorité, beaucoup d'abolitionnistes n'ayant

(1) Professeur d'Histoire contemporaine.

(2) La Hollande a aboli la peine de mort en 1870, l'Italie en 1889 ; en Belgique, la peine de mort est maintenue dans le code pénal, mais on ne l'applique plus depuis 1869 (Jean Imbert, La peine de mort, Paris, PUF, 1989).

(3) Journal officiel,

Chambre des députés, séances des 3 et 7 juillet, 4, 11 et 18 novembre, 7 et 8 décembre 1908 . D'importants extraits du débat ont été publiés par la Société d'études jaurésiennes : "Abolir la peine de mort. Le débat parlementaire de 1908”, Cabier Jaurès nº 2, 1992.

(4) Robert NYE, Crime, Madness and

Gérard Ball / pp. 113 à 126 
Politics in modern France,

Princeton University

Press, 1984.

(5) Dominique KALI-

FA, L'encre et le sang.

Récits de crimes et société à la Belle Époque, Paris, Fayard, 1995.

(6) La Petite République, 27 septembre 1907.

(7) Lois de 1832 sur les circonstances atténuantes, de 1885 sur la libération conditionnelle, de 1891 sur le sursis.

(8) Robert BADIN-

TER, La prison républicaine, Paris, Fayard, 1992, p. 254.

(9) "L'infâme satyre ", dit le socialiste Willm dans la séance $d u$ 4 novembre, a donné à la peine de mort " une virginité nouvelle ».

La peine de mort était "virtuellement abolie ", écrit le radical Lucien Victor-Meunier dans Les Annales de la jeunesse lä̈que (octobre 1907). L'affaire Soleilland va " détruire le labeur de tout un siècle». pas voulu paraître renier leurs convictions. L'abolition semble venir à son heure et couronner un long processus d'adoucissement des peines. ${ }^{(7)}$ L'effectif des détenus dans les prisons a diminué de $40 \%$ de 1893 à 1907 et l'indulgence n'a pas empêché le recul de la criminalité. ${ }^{(8)}$

Mais les dispositions de la Chambre vont se retourner. La commission des réformes judiciaires, qui avait publié un premier rapport favorable au projet gouvernemental en octobre 1907, en publie un nouveau en sens contraire en juin 1908. Le débat s'engage au Palais Bourbon en juillet 1908, il reprend en novembre et se conclut, le 8 décembre, par un vote qui maintient la peine de mort par 330 voix contre 201 . Une cinquantaine de députés qui avaient voté la suppression des crédits du bourreau en 1906, essentiellement des radicaux, ont changé d'avis. La minorité abolitionniste comprend tous les socialistes, la majorité des radicauxsocialistes et quelques députés de la Gauche radicale, une petite poignée de "républicains de gauche" (modérés). La Chambre a cédé à un mouvement d'opinion. Un crime répugnant a joué un rôle essentiel : en janvier 1907, Soleilland ${ }^{(9)}$ a violé, tué et dépecé une enfant de onze ans ; condamné à mort en juillet, il est gracié en septembre, ce qui déclenche des manifestations hostiles à Fallières sur les boulevards. Cette affaire vient renforcer la campagne sécuritaire menée depuis plusieurs années par la grande presse qui se sert de l'inflation du fait divers criminel pour fidéliser sa clientèle. Les lecteurs sont tenus en haleine par la chronique des méfaits des "chauffeurs de la Drôme ", des "bandits d'Hazebrouck ", ou par celle des exploits des " apaches » parisiens, auxquels l'affaire Casque d'or, en 1902, a assuré une douteuse célébrité. L'image qui ressort de ces récits est celle d'une société assiégée par "l'armée du crime ». De fait, le nombre des homicides augmente depuis 1903. Cette recrudescence est attribuée par la presse à " la crise de la répression ", au laxisme de la justice qui abuse du sursis, des circonstances atténuantes, de la libération conditionnelle, qui n'applique pas la loi de 1885 sur la relégation des récidivistes, et bien sûr au chômage de l'échafaud. ${ }^{(10)}$ Les jurys se remettent à condamner à mort ${ }^{(11)}$; ils pétitionnent contre le projet d'abolition et les conseils généraux adoptent des résolutions qui vont dans le même sens. La presse exploite le filon : après la grâce de Soleilland, Le Petit Parisien 
organise parmi ses lecteurs un référendum agrémenté d'un concours de cartes postales ; plus d'un million de lecteurs plébiscitent la peine de mort, contre quelque trois cent mille abolitionnistes.

\section{"Une peine préservatrice pour la société ${ }^{(12)}$ »}

Le débat parlementaire est passionné, chargé de réminiscences historiques (les morts de la Terreur, victimes de l'abolitionniste Robespierre ; les Communards fusilleurs d'otages ; les supplices de l'Ancien Régime dont les tenants de l'exemplarité de la peine capitale devraient demander le rétablissement s'ils étaient conséquents... $\left.{ }^{(13)}\right)$ Il comprend néanmoins toute une partie "technique ", "objective ", qui porte sur les statistiques de la criminalité, sur les conséquences de l'abolition dans les pays voisins ; on peut y rattacher les discussions classiques sur le caractère dissuasif de la peine capitale, sur le risque d'erreur judiciaire, sur la nature des peines à substituer à la mort. Le reste du débat, qui relève des principes, porte sur le droit de la société à infliger la mort, sur la part des responsabilités sociales et individuelles dans la criminalité.

Le silence de Clemenceau pourrait étonner. Sans doute ne désire-t-il pas s'opposer aux éléments les plus modérés de sa majorité. Il n'en est pas moins présent dans la discussion car les socialistes font un grand usage de ses articles abolitionnistes des années quatre-vingt-dix. Les principaux avocats de la peine capitale sont le rapporteur de la commission, Castillard ${ }^{(14)}$ (pour la presse abolitionniste, la guillotine est la " castillarde »...), des hommes de droite comme Barrès, Georges Berry, député nationaliste de Paris, Jules Dansette, député catholique d'Armentières, le président radical-socialiste de la commission, Puech (il est de ces radicaux qui ont changé d'avis depuis 1906), les modérés Failliot et de Folleville ; l'ancien avocat de Dreyfus, Labori, leur apporte un concours quelque peu inattendu. Les champions de l'abolition, outre le garde des Sceaux Briand, sont les socialistes Jaurès, Sembat, Willm, Allemane, Dejeante, les radicaux-socialistes ou radicaux Paul Meunier, Thierry Cazes, Tenting, le "bon juge » Magnaud, ${ }^{(15)}$ les modérés Joseph Reinach et Deschanel ; l'abbé Lemire, habitué à recueillir plus d'applaudissements à gauche que parmi ses coreligionnaires, est le seul
(10) Voir un dessin publié le 8 juillet 1908 par Le Figaro. Le juge à l'accusé : «Vous me copierez trois fois : "J'ai tué Mme Lebon et ses deux filles" "...

(11) Jamais plus de vingt condamnations par an entre 1897 et 1905 , vingt-cinq en 1906, quarante-et-une en 1907.

(12) Georges Berry, 4 novembre.

(13) Le radical-socialiste Paul Meunier donne lecture du récit du supplice de Damiens (4 novembre).

(14) Député de l'Aube, ancien procureur de la République, inscrit au groupe modéré de l'Union démocratique. Élu sénateur en 1909, il siège à la Gauche démocratique et figure jusqu'en 1911 sur la liste des parlementaires membres du parti radical.

(15) Député de la Seine, ancien président du tribunal de ChâteauThierry, célèbre à gauche 
pour son indulgence de principe pour les victimes de l'injustice sociale.

(16) 4 novembre.

(17) Ne voter ni le projet du gouvernement, qui est prématuré, ni le texte de la commission qui « ne va pas dans le sens de la tradition républicaine » en rendant à la peine de mort " une sorte de virginité »; se contenter de mettre en place d'autres peines qui se substitueront à la peine de mort quand elles auront prouvé leur efficacité (7 décembre).

(18) Reinach, 3 juillet.

(19) Briand, 11 novembre.

(20) Magnaud, 7 décembre.

(21) Maurice Ajam, 3 juillet.

(22) 4 novembre.

(23) 11 novembre.

Berry a déposé en octobre 1908 une proposition de loi étendant les cas d'application de la peine capitale.

(24) 3 juillet. catholique abolitionniste. Tous ont conscience de parler sous la pression de l'opinion : "Nous avons le devoir d'écouter la voix du peuple ", affirme Berry, ${ }^{(16)}$ et le radical Gérard-Varet, qui veut défendre une voie moyenne, ${ }^{(17)}$ évoque "le frisson de l'instinct national ». Les abolitionnistes reconnaissent que "le vent a tourné » ${ }^{(18)}$ mais rappellent que le devoir des élus est de se faire les "éducateurs ", non les " esclaves " de l'opinion. ${ }^{(19)} \mathrm{La}$ foule a acclamé Napoléon III, Boulanger, elle a "burlé à la mort » contre Zola, Picquart et Clemenceau lorsqu'ils défendaient Dreyfus...(20)

Les chiffres sont abondamment sollicités, même si la formation humaniste des députés les incite au scepticisme ("La statistique est une bonne fille, elle va avec celui qui la caresse le mieux $»{ }^{(21)}$ ) Les abolitionnistes n'ont pas de mal à démontrer que l'arrêt des exécutions en Belgique ou en Italie n'a pas inversé la tendance séculaire au recul de la criminalité. On leur objecte la recrudescence récente des homicides en France : "On exécute moins", résume Labori, "immédiatement, le nombre des crimes de sang augmente ". ${ }^{(22)}$ Le débat sur les chiffres est quelque peu répétitif et confus, beaucoup de députés ne comprenant pas toujours si l'on parle de l'ensemble des crimes commis en une année ou seulement de ceux qui ont été jugés. Surtout, la distinction entre l'assassinat et le meurtre semble mal passer dans cette assemblée qui compte pourtant de nombreux juristes. Briand ne nie pas qu'il y ait davantage d'homicides, mais le nombre des assassinats légalement passibles de la peine de mort n'a pas crû dans la même proportion. La reprise des exécutions capitales n'aurait pas d'effet sur les "crimes d'impulsion". Ce que conteste évidemment l'autre camp, qui s'indigne de voir le ministre considérer les crimes des apaches comme " de simples meurtres sans importance " (Berry) et qui créditent la peine de mort d'un effet dissuasif global : "Pour la masse du peuple ", affirme Castillard, " ce qui entraîne la peine de mort, c'est le fait de tuer son semblable, c'est le fait de jouer du couteau, de jouer du revolver, de tuer son homme ». ${ }^{(23)}$

La réfutation de l'exemplarité de la peine de mort est un thème classique depuis Beccaria. Mais Joseph Reinach est sans doute trop optimiste lorsqu'il estime que "personne n'ose plus dire comme Joseph de Maistre que le bourreau est le lien de l'association sociale ${ }^{(24)} "$, car c'est bien une conviction de cet ordre qu'expriment certaines interventions : "la peur de 
l'échafaud est le commencement de la sagesse "; la peine de mort est "une peine préservatrice de la société(25) ». Un argument a beaucoup de succès : la mort est la seule sanction prévue par les lois officieuses qui régissent les associations de malfaiteurs. ${ }^{(26)}$ Les abolitionnistes utilisent les nombreux témoignages montrant que bien des assassins ont déjà eu l'occasion d'assister à une exécution capitale et que ce spectacle ne leur a pas inspiré un effroi salutaire, mais plutôt un "mysticisme malsain ", la " griserie $d u$ sang ${ }^{(27)} \mathrm{Au}$ reste, tous les intervenants s'accordent pour réclamer la fin des exécutions publiques (elles continueront pourtant jusqu'en 1939), qui éveillent une curiosité morbide et provoquent des désordres scandaleux, qui font surtout au criminel l'honneur d'une " mort de première classe ", d'une "apothéose infâme " de nature à lui susciter des émules. ${ }^{(28)}$ Il n'est pas difficile aux abolitionnistes, dans ces conditions, d'ironiser sur l'exemplarité d'une peine que la décence comme la prudence conseillent de dissimuler, comme dit Sembat, "dans les caves et les catacombes " : "si la peine de mort est une chose exemplaire, il faut l'étaler au grand soleil ${ }^{(29)} " \ldots$

Le caractère irréparable de la peine de mort est un autre thème traditionnel. Pour Deschanel, c'est l'argument essentiel : "Il suffit qu'au cours des siècles un seul homme ait été injustement condamné à la peine capitale pour que la peine capitale doive disparaître. " L'échafaud est un « monument bideux de l'orgueil bumain, puisqu'il atteste la croyance naïve de l'homme en son infaillibilité( ${ }^{(30)} »$. L'affaire Dreyfus est évidemment sollicitée pour rappeler la fragilité des décisions de justice, mais aussi les condamnations de Calas et de Sirven, et même les procès de Socrate et de Jésus. L'erreur peut aussi consister à exécuter un fou. ${ }^{(31)}$ Les défenseurs de la guillotine répondent que le risque d'erreur définitive est considérablement atténué par la possibilité des circonstances atténuantes et de la grâce. Au reste, ils semblent s'en accommoder avec une sorte de résignation fataliste. Il y a beaucoup plus d'erreurs médicales que d'erreurs judiciaires, assure Castillard. ${ }^{(32)}$ Et Labori, à qui l'on fait remarquer que son client Dreyfus aurait pu être fusillé, répond qu'on aurait encore pu se battre pour réhabiliter sa mémoire : "N'y a-t-il pas des choses, et notamment l'bonneur, qui sont supérieures à l'existence ?(33) ${ }^{(3)}$ La question de la peine de remplacement
(25) Failliot, 3 juillet ; Berry, 4 novembre.

(26 Ajam, 3 juillet ; Labori, 4 novembre. Briand objecte que les apaches ne disposent pas de prisons, et qu'il n'est guère flatteur pour la société de calquer ses lois sur celles des criminels (11 novembre).

(27) Briand,

11 novembre.

(28) Failliot, Barrès, 3 juillet.

(29) 4 novembre.

(30) 4 novembre.

(31) Willm rappelle que l'autopsie de l'éventreur Vacher, exécuté en 1898, a montré qu'il avait une balle dans la tête. " Bah, aurait déclaré le juge, on ne peut pourtant pas disséquer les condamnés à mort avant de les exécuter » (4 novembre). Castillard luimême, révèle Briand, aurait dit en commission qu'il n'aurait sans doute pas envoyé Soleilland à la guillotine (11 novembre). 
(32) 11 novembre.

(33) 4 novembre.

(34) 7 décembre.

(35) 4 novembre.

(36) Il s'en prend aux passe-droits qui permettent aux "fils de famille " envoyés au bagne de devenir gratte-papier ou même "nourrices sèches".

(37) 7 décembre.

(38) 7 décembre.

(39) En Italie, affirme Jaurès, les criminels ont très peur de l'ergastolo.

"Cela prouve que même des imaginations méridionales peuvent se représenter avec une vivacité suffisante une autre peine que la peine de mort" (18 novembre).

(40) Le Radical,

3 novembre 1908 .

(41) Paul Meunier, 4 novembre. occupe une place importante dans le débat, car la plupart des députés semblent estimer que, peine de mort mise à part, les pénalités existantes ne sont pas suffisamment dissuasives. Même le "bon juge" Magnaud considère qu' "il faut substituer à la peine de mort une peine plus effrayante pour le criminel que la peine de mort elle-même ${ }^{(34)} »$. Deschanel dénonce le laxisme judiciaire : "La répression ne doit pas être affaiblie outre mesure, énervée par l'abus des sursis et des libérations conditionnelles et par le relâchement du régime de la transportation. ${ }^{(35)}$ "Allemane, qui a connu le bagne de Nouvelle Calédonie après la Commune, est le seul à estimer que "les peines existantes sont déjà trop épouvantables ${ }^{(36)}$ ", et le seul abolitionniste à s'élever contre la perspective de l'encellulement, qui ferait sombrer les condamnés dans la folie ${ }^{(37)}$ (ce que disent aussi les partisans de la guillotine, avec un zèle humanitaire suspect). Les autres orateurs paraissent juger que ni la perspective de la prison, ni celle du bagne ne sont de nature à effrayer les criminels. Le "confort" des prisons de la République, prétend le radical Gérard-Varet, "se dresse comme une douloureuse ironie en face de la misère honnête et vaillante ${ }^{(38)}$ ". D'ailleurs, on s'évade trop facilement de la prison ou du bagne, ou tout au moins on s'imagine qu'on pourra s'en évader (Briand doit rappeler que la plupart des évadés du bagne sont repris ou meurent pendant leur fuite). Pour Gérard-Varet, les criminels perçoivent plus le bagne comme une sorte de destination touristique que comme un lieu d'expiation : "Faillite $d u$ bagne, avec cette sorte de prestige qui s'attache aux voyages lointains, aux pays ensoleillés, aux grands océans, aux continents mystérieux, avec le mirage des évasions possibles toujours, réelles souvent. " Paradoxalement, ce sont les "humanitaires" qui doivent assurer que le bagne, la prison ou l'encellulement sont des châtiments terribles...(39)

\section{"La formule intangible du parti républicain ${ }^{(40)}$ "}

Le cœur du débat, c'est la revue des principes. Les abolitionnistes entendent montrer que l'abolition, aboutissement du mouvement d'adoucissement des peines qui a pris naissance au siècle des Lumières, s'inscrit dans une tradition humaniste de progrès que la France se doit de respecter, elle qui a "la réputation de marcher à la tête de la civilisation ${ }^{(41)}$ ". 
La peine de mort est du côté de la régression, de l'archaïsme, de la vengeance primitive, du talion. Clemenceau a développé cet argument de façon frappante dans des pages de La Mêlée sociale (1895) et du Grand Pan (1896) citées par Willm : "Le sang est une vieille pâture des ancêtres dont le goût nous monte aux lèvres dès qu'on le présente à nos yeux. " "Depuis l'anthropoïde à long bras, l’homme continue, en la tempérant plus ou moins, l'œuvre de meurtre imposée à sa race par un atavisme qu'il n'a pas encore réussi à dompter. " "Tuez donc, mais ne vous donnez pas l'apparence d'être la justice quand vous n'êtes que la vengeance et la férocité, le prolongement héréditaire d'un atavisme de sang... ". Au moment où la politique du gouvernement Clemenceau divise gravement les socialistes et les radicaux, les abolitionnistes rappellent que leur cause appartient au patrimoine indivis de la gauche. L'abolition est "la formule intangible du parti républicain ", vient d'écrire le quotidien gouvernemental Le Radical, cité par Willm. En renonçant à "l'intégrité de son principe ", avertit Jaurès, la gauche permet aux cléricaux de prétendre qu' une société qui a chassé Dieu [...] n'a plus d'autre ressource que le bourreau ${ }^{(42)}$ ". La généalogie républicaine de la réforme commence avec Lepelletier de Saint-Fargeau et Robespierre ${ }^{(43)}$ en 1791, avec la Convention décrétant en 1795 l'abolition « à dater du jour de la publication de la paix générale "; elle se poursuit avec les hommes de 1848, Lamartine, Quinet, Schœlcher, Louis Blanc, Victor Hugo (omniprésent dans le débat, ${ }^{(4)}$ avec les républicains du Corps législatif de 1870, Jules Simon, Gambetta, Ferry. Deux temps forts dans cette mobilisation des grands ancêtres et des grands principes : la lecture par le radical-socialiste Thierry Cazes de la lettre d'un de ses électeurs, "un vénérable paysan du Gers " qui signe " un vieux Montagnard de 1848 ", remplie de citations de Victor Hugo ${ }^{(45)}$; et surtout l'incident provoqué par Barrès pendant le discours de Joseph Reinach. Ce dernier ayant qualifié l'alcoolisme de "fléau destructeur de la race ", Barrès l'interrompt pour suggérer que la seule race qu'il ait compétence pour défendre est la race juive. D’où une solennelle mise au point du président de la Chambre, Henri Brisson : "Ici, nous ne connaissons pas de races. Il n'y a dans cette assemblée que des représentants de la nation française. " "Après cet incident, commente le radical Lagasse, il n'y a pas de républicain qui puisse voter le maintien de la peine de mort. ${ }^{(46)}{ }$ "
(42) 18 novembre.

(43) Un nom dont la mention par Willm et Jaurès provoque évidemment des « murmures » et des « sourires sur divers bancs ». Les socialistes excusent la Terreur par l'habituelle théorie des circonstances : "Ceque vous oubliez, lorsque vous souriez à l'évocation de Robespierre, c'est que ni les uns ni les autres nous ne serions ici sans la Révolution, et sans la Révolution telle qu'elle s'est comportée par suite de nécessités intérieures et extérieures» (Willm, 3 juillet).

(44) « En vous entendant, je vous reconnais, monsieur Jaurès ; vous vous appelez Mgr Myriel. Mais l'évêque des Misérables de Victor Hugo ne veut même pas que le coupable aille au bagne. Il est tout miséricorde ; il pardonne toujours car il espère toujours le repentir, le retour du coupable. Voilà l'extrémité d'une pensée évangé- 
lique. Je la salue. Mais l'Évangile et l'Église laissent la société se défendre " (Barrès, 18 novembre).

(45) 7 décembre.

(46) 3 juillet.

(47) L'autre prêtre député, l'abbé Gayraud, s'abstiendra. Pendant la discussion, il a marqué son désaccord avec les vues de Barrès sur la déchéance irrémédiable du criminel (3 juillet). Il proteste également lorsque Labori déclare que les « mystiques » qui croient à la vie éternelle devraient accepter facilement de "briser une vie si c'est dans l'intérêt social et pour une cause juste » (4 novembre).

(48) 18 novembre.

(49) 3 juillet.
Jaurès prononce un discours audacieusement œcuménique, qui invoque contre la peine de mort à la fois l'esprit de la Révolution et celui du christianisme. La peine de mort est "un dogme de fatalité » en vertu duquel il existe "des individus irrémédiablement tarés", comme il y a "des races socialement, historiquement maudites" et "des classes socialement mandites». C'est sur ce «bloc de fatalités » que se dresse l'échafaud. Or la Révolution, c'est "le refus de la fatalité", c'est "une magnifique affirmation de confiance dans la nature humaine en elle-même ». Par une étonnante métaphore ferroviaire, la guillotine devient le symbole du refus du progrès : "Elle a pour mission de signifier aux hommes que jamais le progrès social, jamais le progrès de l'éducation et de la justice ne dispensera les sociétés bumaines de tuer et de répondre à la violence individuelle par le meurtre sanglant [...] C'est le disque rouge projetant ses lueurs sanglantes sur les rails et signifiant que la voie est barrée, que l'espérance humaine ne passera pas. "

Mais ce "désespoir systématique et éternel " est aussi en contradiction avec le christianisme. Jaurès donne une leçon de théologie aux députés catholiques qui, à l'exception de l'abbé Lemire, voteront pour la peine de mort. ${ }^{(47)}$ Avec les deux dogmes du péché originel et de la rédemption, le christianisme " a été tout ensemble une grande prédication d'bumilité et de confiance. Il a proclamé, avec l'universelle chute, l'universelle possibilité du relèvement. " La présence de l'aumônier qui assiste le condamné ne serait qu'une "comédie lugubre » si elle ne signifiait pas qu'on reconnaît chez le criminel "la vocation au relèvement, la possibilité du relèvement ${ }^{(48)}$ ». Tout ceci répond à Barrès qui soutient que la société doit éliminer les "dégénérés", les "éléments antisociaux " (et quant à la présence du prêtre, elle signifie seulement "qu'ily a en effet de grandes difficultés qui peuvent être résolues dans l'autre monde $\left.{ }^{(49)} "\right)$.

L'abbé Lemire, très applaudi à gauche, ne contredit pas l'argumentation de Jaurès, tout en précisant que son intention n'était pas de parler en chrétien, mais « comme homme et comme Français », attaché aux « droits de l'bomme que les principes modernes ont reconnus à tous". Lui aussi rejette le "fatalisme " et le " matérialisme " du " dogme du bourreau obligatoire". "Je demande, au nom des droits de l'bumanité, que vous respectiez la personne, que vous lui donniez le temps de se raisonner, de se ressaisir et de traîner sa honte, 
de sentir son remords accablant jusqu'à la tombe [...] A la place d'une société dominée par le sinistre échafaud sanglant, je voudrais une société couronnée par la possibilité indéfinie du remords, du repentir et de l'expiation. ${ }^{50)}$ "

Il ne faut pas voir dans cette apologie de la pénitence un trait spécifiquement chrétien, car les socialistes et les radicaux ne disent guère autre chose. Jaurès veut que les criminels continuent de vivre pour qu'ils aient "le loisir de penser et de réfléchir ». Le vieux paysan quarante-huitard cité par Thierry Cazes se réfère à Victor Hugo et à l'œil qui regarde Caïn dans sa tombe pour affirmer que "le châtiment le plus effrayant pour le criminel, ce n'est pas la mort mais le cri de la conscience ". Et Lemire fait applaudir par les libres penseurs sa synthèse de l'esprit chrétien et de l'idéal démocratique : la démocratie, au nom de la dignité humaine, exige que l'homme puisse " consentir à toutes les exigences et à toutes les conditions sociales ", à la loi comme à l'impôt. "Logiquement nous devons arriver à ce que l'homme puisse aussi donner son consentement à la peine qu'il subit ", car la plus belle victoire " consiste à triompher du mal dans une conscience bumaine et à lui faire accepter la justice ".

Que peuvent répondre les partisans de la peine de mort à ces effusions d'idéalisme laïque et chrétien ? Barrès est le seul à rejeter catégoriquement les postulats optimistes qui les inspirent. Les abolitionnistes se trompent lorsqu'ils se représentent le criminel comme un être fruste, un "barbare » éducable ("Si vous lui aviez donné le livre », disait Hugo, "vous auriez détruit le crime. ${ }^{(51)}$ ") La " science " montre au contraire que le meurtrier n'est pas un "être trop neuf " mais un "dégénéré ", "entravé par des tares ignobles ", "un homme tombé en debors de l'bumanité et non pas un bomme qui n'est pas encore arrivé à l'bumanité". Il y a là un écho des vues de Lombroso sur le criminel-né. Rappelons que la préface de Taine à l'édition française de L'Homme criminel, publiée en 1887, qualifiait les assassins d' "orangs-outangs à face bumaine » et jugeait "d'autant plus nécessaire de les éliminer qu'ils sont et resteront des orangs-outangs"...

Barrès voit dans l'indulgence des " gens trop cultivés" pour les criminels le symptôme d'une "maladie de l'intelligence ", d'un "affaiblissement de la volonté ", d'une peur de prendre la responsabilité de juger. A cette dangereuse contagion du tolstoïsme, il oppose l'exemple de "virilité sociale »
(50) 18 novembre.

(51) C'est encore le point de vue des Annales de la Jeunesse lä̈que : «Il n'est pas permis de considérer les criminels comme des machines mal faites ou des êtres ne relevant que de la douche ou du bromure de potassium. Ce sont des consciences ignorantes, inférieures, obscures; il faut les améliorer, les redresser, les guérir et non les supprimer " (Évariste Giaterski, juin 1906). 
(52) 3 juillet.

(53) De Folleville, 7 décembre.

(54) Gioux, 7 décembre.

(55) 7 décembre.

(56) 4 novembre.

(57) Ajam, 3 juillet.

(58) 4 novembre. que donne l'Angleterre où "les jeunes gens des sociétés de gymnastique " sont allés " tuer quelques apaches " dans les rues de Londre... ${ }^{(52)}$

Les autres adversaires de l'abolition sont plus prudents, même s'ils ironisent eux aussi sur "l'bumanitairerie " (Berry), le "bluff humanitaire " (Castillard), "la sensibilité maladive qui est à la société ce que la neurasthénie est aux individus " (Dansette). Ils ne nient pas que l'abolition soit moralement souhaitable et qu'on y parviendra sans doute un jour. Mais il ne faut pas " mettre la charrue devant les boufs ${ }^{(53)}$ "; il faut d'abord enrayer la criminalité, rassurer l'opinion, mettre en place des peines alternatives qui aient le temps de faire leurs preuves. Le disque rouge dont parlait Jaurès permet d'établir "une zone de sécurité à l'abri de laquelle la société pourra poursuivre en paix son évolution progressive ${ }^{(54)}$ ". Cet état d'esprit est caractéristique des députés radicaux qui ont fait marche arrière depuis 1906 ; ce sont des " abolitionnistes honteux ", s'indigne Tenting, des "opportunistes de l'échafaud ${ }^{(55)}$ ». Labori reconnaît que la peine de mort lui "répugne ", que l'abolition est "la formule de l'avenir ". Mais, " dans la situation sociale actuelle ", la peine de mort est un moindre mal (tout comme la guerre, que tout le monde souhaiterait voir disparaître). ${ }^{(56)}$ "Ma doctrine philosophique personnelle est dans le sens de l'abolition ", déclare le radical Maurice Ajam. Mais "à l'heure actuelle, dans le pays, cette opinion philosophique n'a pas eu sa répercussion". Il propose d'ailleurs une solution transactionnelle : on n'abolirait pas la peine de mort, mais on inscrirait dans les codes, comme peine équivalente, l'internement perpétuel en laissant au jury le choix entre la peine "archaïque et empirique » et " une peine plus moderne ». Bientôt, l'évolution des esprits permettrait de " guillotiner la guillotine ${ }^{(57)}$ ".

\section{La question des causes de la criminalité}

Tout ce qui précède suggère la diversité des visions du criminel. L’armée du crime est hétérogène : brutes irrémédiablement dégénérées, calculateurs cyniques, barbares voués au meurtre par un déficit d'éducation morale ou d'éducation tout court, impulsifs incapables de résister à leurs instincts, dilettantes du crime... Le crime, déclare Sembat, est "à la fois un produit individuel et un produit social ${ }^{(58)}$ ", et les abolitionnistes 
font évidemment une grande place dans leur argumentation aux facteurs sociaux de la criminalité. Dès 1885, au congrès d'anthropologie criminelle de Rome, Alexandre Lacassagne, professeur de médecine légale à Lyon, s'opposait à la thèse de Lombroso sur la fatalité biologique du crime en affirmant que "les sociétés ont les criminels qu'elles méritent ", que le milieu social agit sur le criminel potentiel comme le bouillon de culture sur le microbe. ${ }^{(59)}$

Tout le monde ou presque déplore les méfaits de l'alcoolisme, ${ }^{(60)} \mathrm{de}$ "l'effrayante multiplicité des cabarets ${ }^{(61)}$ ", encore que certains députés tiennent à préciser que les eaux-de-vie de leur terroir n'ont rien à voir avec l'alcool industriel servi dans les assommoirs urbains. ${ }^{(62)}$ Mais le Parlement vient, en 1905, de couper court aux tentatives de limitation du privilège des bouilleurs de cru et, en 1911, la Chambre va rejeter un projet de limitation des débits de boisson adopté par le Sénat... ${ }^{(3)}$

Le rôle criminogène de la grande presse d'information, de l'héroïsation malsaine des exploits des apaches, est également un thème plutôt consensuel. Certains journaux sont "de véritables écoles $d u$ crime », déplore Reinach qui dénonce la collaboration trop fréquente entre magistrats, policiers et journalistes. ${ }^{\left({ }^{64)}\right.}$ Pour Allemane, ce sont les défenseurs des privilèges bourgeois qui ont intérêt à ce que la chronique criminelle accapare l'attention du public: Mme Steinheil semble «avoir été mise en location pour occuper l'opinion publique ${ }^{(65)}$ ». Gérard-Varet évoque le sabotage de l'œuvre moralisatrice de l'école par les journaux en des termes annonciateurs de nos modernes polémiques sur le rôle de la télévision : "La société s'est habituée à demander tout à l'école ", mais cette même société "fait tout contre l'école » en laissant le public s'abreuver «d'un flot d'images violentes et troublantes, d'émotions fascinatrices". Il conclut de ce "détraquement de la conscience collective » que la peine de mort ne pourra disparaître que lorsque le peuple français aura retrouvé la «santé morale ${ }^{(66)}$ ». Notons, à propos de l'école, que les députés conservateurs, en dehors de quelques interruptions, n'abusent pas de la polémique classique contre l'école sans Dieu, école de l'immoralité et du crime. ${ }^{(67)}$ Lemire peut même inviter les libres penseurs et les croyants à unir leurs efforts pour " garantir les éternels fondements du droit ».

(59) Mais son livre de 1908, Peine de mort et criminalité, conclut à la nécessité du maintien de la peine de mort.

(60) Clemenceau ne sort de son mutisme que pour approuver de son banc, le 11 novembre, Briand qui rappelle l'urgence du vote d'une loi sur la limitation du nombre des débits de boisson.

(61) Lemire, 18 novembre.

(62) « Laissez boire le bon alcool et supprimez le mauvais " (Lasies, député du Gers, 11 novembre). Jaurès ayant évoqué les " hommes à la lèvre pendante, à l'œil bébété" qu'on peut rencontrer dans les fermes normandes, il est interrompu par le député du Calvados, Henri Laniel :

"L'alcool exerce les pires ravages dans les grandes villes, où l'on ne consomme que de l'alcool industriel. Allez voir nos conseils de révision! Un grand nombre 
de nos jeunes gens sont envoyés dans les troupes d'élite, dans l'artillerie notamment "

(18 novembre).

(63) Voir Didier

Nourrisson, Le buveur du XIXème siècle, Paris, Albin Michel, 1990, pp. 278-285.

(64) 3 juillet. Briand déclare qu'il a donné des instructions pour la faire cesser.

(65) 7 décembre. Sur l'affaire Steinheil, contemporaine du débat de 1908, cf. Pierre

Darmon, Marguerite Steinheil, Ingénue criminelle ?, Paris, Perrin, 1996.

(66) 7 décembre.

(67) Quand Reinach rappelle que «la criminalité est surtout une criminalité de jeunes gens », Suchetet interrompt pour préciser que « ce sont des jeunes gens formés par l'école neutre » (3 juillet).

(68) Willm, 3 juillet. (69) 7 décembre.
Les abolitionnistes font une peinture véhémente des fléaux sociaux, misère, bas salaires, prostitution, chômage, errance, abandon de l'enfance, qui peuvent faire basculer les déshérités dans la criminalité. "Ce sont les conditions économiques qui sont les seules causes profondes, certaines et réelles de la criminalité [...] Si, au lieu de perdre notre temps en querelles stériles, nous nous occupions un peu plus des déshérités qui souffrent et qui ont besoin d'être protégés, si nous faisions des lois sociales, vous verriez immédiatement diminuer la criminalité. ${ }^{(68)}$ » Inévitable et facile polémique socialiste contre un gouvernement radical accusé de s'accommoder trop aisément de l'ajournement des réformes inscrites à son programme ? Mais le Clemenceau de La Mêlée sociale, évidemment cité par Willm, ne parlait pas autrement : "Un grand cri part de la foule irresponsable : Tue! Et le gouvernement ne résiste à la foule que lorsqu'elle demande la réforme des abus. Pourtant, plus de pitié des misérables, fatalement, aboutirait à moins de besogne pour le bourreau. " Il y a aussi des "crimes de riches", objecte de Folleville... ${ }^{(69)}$ Mais Jaurès, sans nier "la part des responsabilités individuelles qui s'ajoutent à la responsabilité sociale ", soutient que la psychologie du criminel, dominée par "l'insensibilité et l'orgueil ", est façonnée par "la vie d'abandon où il est laissé ». "Ce qui doit être l'objection principale contre la peine de mort, c'est qu'elle détourne de la recherche des responsabilités sociales dans le crime. ${ }^{(70)}$ ". Et Jaurès d'invoquer, de façon quelque peu inattendue, la philosophie sociale quasi-officielle de la République, le solidarisme ${ }^{(71)}$ : "C'est trop commode de créer ainsi un abîme entre les coupables et les innocents. Il y a des uns aux autres une chaîne de responsabilités. Il y a une part de solidarité. Nous sommes solidaires de tous les hommes, même dans le crime [...] C'est pour détourner la part des responsabilités sociales qu'on essaie de dresser l'échafaud; et nous, pour préparer la solidarité sociale, nous voulons la justice dans la paix. ${ }^{(72)}{ }^{\star}$

"L'abolition a été une grande cause de la gauche française », déclarait Robert Badinter devant l'Assemblée nationale le 17 septembre 1981, à l'ouverture du débat qui allait, cette fois, enterrer la guillotine. La formule était encore plus exacte en 1908 puisqu'en 1981 un nombre non négligeable de parlementaires de droite avait rejoint le camp abolitionniste. L'échec de 1908 est donc celui de toute une tradition progressiste, humaniste, 
" bumanitaire ", comme on disait à l'époque ; c'est l'échec du républicanisme utopique à la Victor Hugo.

Et cet échec n'est pas un accident isolé. Il est à mettre en rapport avec le virage centriste, modéré de la République, qu'on avait pu croire réancrée à gauche au lendemain de l'affaire Dreyfus. Pendant les batailles laïques du Bloc, les républicains anticléricaux croyaient au "postulat combiste " : la République, définitivement consolidée, allait reprendre à une allure accélérée sa marche vers le progrès, vers la démocratie sociale. Délivrons les intelligences du "joug romain ", écrivait Clemenceau en 1904, et "l'esprit humain pourra librement reprendre son essor dans les voies oubliées de la grande Révolution ${ }^{(73)}$ ». Mais, après le triomphe électoral de 1906, la brouille s'installe entre radicaux et socialistes ; la majorité des radicaux cède à la peur sociale et Clemenceau mène une répression musclée contre le syndicalisme révolutionnaire, les antimilitaristes, les vignerons révoltés du Midi, les fonctionnaires qui revendiquent le droit syndical. Les réformes dont les programmes électoraux proclamaient l'urgence, impôt sur le revenu, retraites ouvrières, n'aboutissent pas, du fait de réticences qui se manifestent à l'intérieur même de la majorité gouvernementale. L'échec de l'abolition de la peine de mort est à rapprocher de celui d'une autre réforme que toute la gauche proclamait indispensable au lendemain de l'affaire Dreyfus, celle de la justice militaire, la suppression des conseils de guerre. ${ }^{(74)}$

L'aspiration de la majorité républicaine à l'ordre et à la sécurité se traduit en crispation autoritaire. L'insécurité, ce ne sont pas seulement les apaches, c'est aussi l'agitation ouvrière et le discours révolutionnaire des chefs de la CGT. C'est aussi, depuis la crise marocaine de 1905, l'appréhension de la guerre. Le pacifisme est également une valeur en baisse dans la gauche gouvernementale. Remarquons que la grande presse n'hésite pas à faire l'amalgame entre le péril de la délinquance et le danger révolutionnaire. Pour Le Petit Parisien, "les apaches suivent la philosophie de l'action directe, mettant en pratique les conseils des penseurs des partis d'avantgarde tels que M. Sorel dans ses Réflexions sur la violence ${ }^{(75)}$ ". "M. Jaurès protège l'apache, c'était immanquable ", écrit Le Matin. ${ }^{(76)}$ Si l'on parcourt la presse radicale en 1907, pendant les troubles du Midi, on y trouve des

(70) Sur ce point encore, le discours de Lemire prolonge celui de Jaurès : dans la brutalité de la peine capitale, il y a « le vague désir de se soustraire au reproche importun qu'est l'assassin vivant ». Car celui-ci "n'est pas un produit isolé, une croissance sans racines. Avant de le frapper, il est juste que le législateur rentre dans sa propre conscience".

(71) De Folleville s'en indigne : "Notre devoir de solidarité doit s'arrêter devant le crime"

(7 décembre).

(72) 18 novembre.

(73) L'Aurore, 20 mars 1904.

(74) Le 25 juin 1907, la Chambre a ajourné le débat (dans un contexte marqué par la mutinerie du 17ème et la peur de la contagion de l'antimilitarisme cégétiste et hervéiste) qui ne s'engage qu'en mars 1909. Le projet adopté le 11 juin 1909 par les députés ne franchira pas l'obstacle 
sénatorial. Dans Les Annales de la jeunesse laïque d'août 1907, Lucien Victor-Meunier rapproche le sort des deux réformes : « $D e$ même que le crime abominable d'un Soleilland a fait renoncer à la suppression de la peine de mort, il a suffi de quelques actes d'insubordination pour faire renoncer à la suppression de cette infâme parodie de justice qu'on appelle la justice militaire ». Le même journaliste voit dans la reprise des exécutions capitales, au début de 1909, le symbole de l'avortement du radicalisme clemenciste : «Ce n'était pas assez que nous vécussions sans progrès, sans réformes, sans action [...], il a fallu encore, lamentable conséquence de la politique lamentable que nous subissons, que nous fissions un pas de côté, en arrière.

Nous revoilà les pieds englués dans la boue et dans le sang. En ce mois de janvier 1909, la guillotine manifestations frappantes d'une inquiétude qui ébranle les fondements de l'optimisme progressiste de la gauche : "De toutes parts ", écrit le sénateur Rivet, "les liens sociaux les plus essentiels se relâchent et se rompent [...] Ce que l'on préconise partout sous le nom d'action directe, c'est l'anarchie des appétits et des fantaisies [...] Il y a dans tous les phénomènes de la vie collective un fond d'égoïsme et de lâcheté qui n'est pas niable. Chacun réclame à grands cris la suppression des obligations qui le gênent. " ${ }^{(77)}$ "Peut-être ", se demande Le Rappel, "a-t-on cru trop facilement qu'une démocratie pouvait vivre sans freins ni règles [...] Peut-être a-t-on fait trop entière confiance aux citoyens que vingtcinq ans d'instruction rationnelle n'ont pas encore préparés suffisamment aux avantages, mais aussi aux périls de la liberté. Il faut à ce pays, pour de longues années encore, une direction positive, un gouvernement qui gouverne, des lois $q u$ 'on applique. ${ }^{(78)} »$ Et pour près de trois-quarts de siècle encore, il lui «faudra» la guillotine...

a été restaurée en France.

En attendant le roi ! disent,

fiers de ce premier succès, les malheureux qui, affamés de servitude, voudraient se donner et nous donner un maître " (ibid. février 1909).

(75) Le Petit Parisien, 7 avril 1907.

(76) 9 octobre 1907.

(77) Le Radical, 22 juin 1907.

(78) 28 juin 1907. 